\title{
CALCIFIED PULMONARY METASTASES FROM TESTICULAR AND OVARIAN TUMOURS \\ A REPORT OF TWO CASES WITH LONG SURVIVAL
}

\author{
BY \\ J. SEMPLE AND L. R. WEST \\ From Hairmyres Hospital, Lanarkshire, and Sully Hospital, Glamorgan
}

(RECEIVED FOR PUBLICATION JULY 22, 1955)

Calcification within multiple lung opacities in a radiograph is usually considered strong evidence in favour of a tuberculous aetiology, but it may occur in metastases from an osteogenic sarcoma (Speed, 1943). The following two cases illustrate that similar calcification may occasionally occur in slowly growing or "quiescent" pulmonary metastases from other tumours.

\section{Case Reports}

CASE 1.-A. H., a journalist who had been asthmatic since childhood, had had a swelling in the right testicle since he was at school. In 1934, at the age of 18 , the right testicle was removed, as the swelling had increased. A histological report of the testicular tumour stated that the portions of tumour showed very marked necrosis, and the appearances were those of an "epithelial tumour of the seminoma type." Unfortunately the tissue is not now available for reexamination.

Following operation he had radium treatment to the region of lymphatic drainage. In 1938 he began to have symptoms of " bronchitis" with blood-stained sputum. A chest radiograph (Fig. 1a) showed " round opacities in both lungs, particularly the right, almost certainly due to metastatic deposits." $\mathrm{He}$ was given a course of deep $x$-ray therapy (entire trunk treated by large anterior and posterior fields, 30 treatments, $1,500 \mathrm{r}$., in a period of six weeks). A repeat radiograph in October, 1938, showed "increase in size of the largest chest deposit in the right lung and a new deposit in the left lung." $\mathrm{He}$ was given a further course of deep $x$-ray therapy (five treatments, total of 500 r.). In April, 1940, a chest radiograph (Fig. 1b) showed "the deposits very slightly larger." His general condition at this time was satisfactory, and he was working.

Radiological examination in February, 1943, showed " the metastases slightly denser," and in 1945 stippled calcification was seen in some of the lesions. In the autumn of 1947 he developed a pain in the right chest and coughed up much brownish fluid. A radiograph (December, 1947) showed "the large deposit in the right lung broken down and forming a cavity with a fluid level." A quantity of thick, pinkish fluid was aspirated from this cavity. He was admitted to a chest surgery unit in June, 1948. Radiography showed well-marked calcification in the lung deposits and a large cavity containing fluid in the apex of the right lower lobe (Figs. 1c and d). As the patient at this time was feeling unwell and having copious expectoration, the "cyst" in the right lower lobe was removed at operation. He made an uneventful recovery and was discharged home three weeks later.

The histological report of the resected "cyst" was as follows :

"This is a teratoma, and the appearances are in agreement with the clinical history of a testicular primary. The tumour is somewhat degenerate, but it still appears to be viable. It is comprised of many elements, the most prominent being lumps of cartilage. There is also a portion of true bone formation, the medullary cavity being occupied by fine fibrous tissue and fat, but no haemopoietic tissue. Small irregular glands are also present and some are cystic ; they are lined by an indeterminate low cubical epithelium and a few contain some narrow stratified squamous epithelium. Smooth muscle fibres are plentiful. This type of secondary teratoma, in which all variety of elements exist, is quite typical of testicular teratoma."

$\mathrm{He}$ was seen at regular intervals during the next four years, and remained well and at work. A chest film taken in July, 1950 (Fig. 1e), showed no change in the remaining partly calcified deposits. In October, 1952, while on holiday, he developed a severe asthmatic paroxysm in which he died. There was no necropsy.

CASE 2.-A mental nurse, a woman aged 29, had previous excellent health. In February, 1950, she complained of lassitude and unproductive cough of two months' duration, and a chest radiograph (Fig. 2a) suggested miliary tuberculosis. She was admitted to a hospital, where she remained afebrile, the sputum 


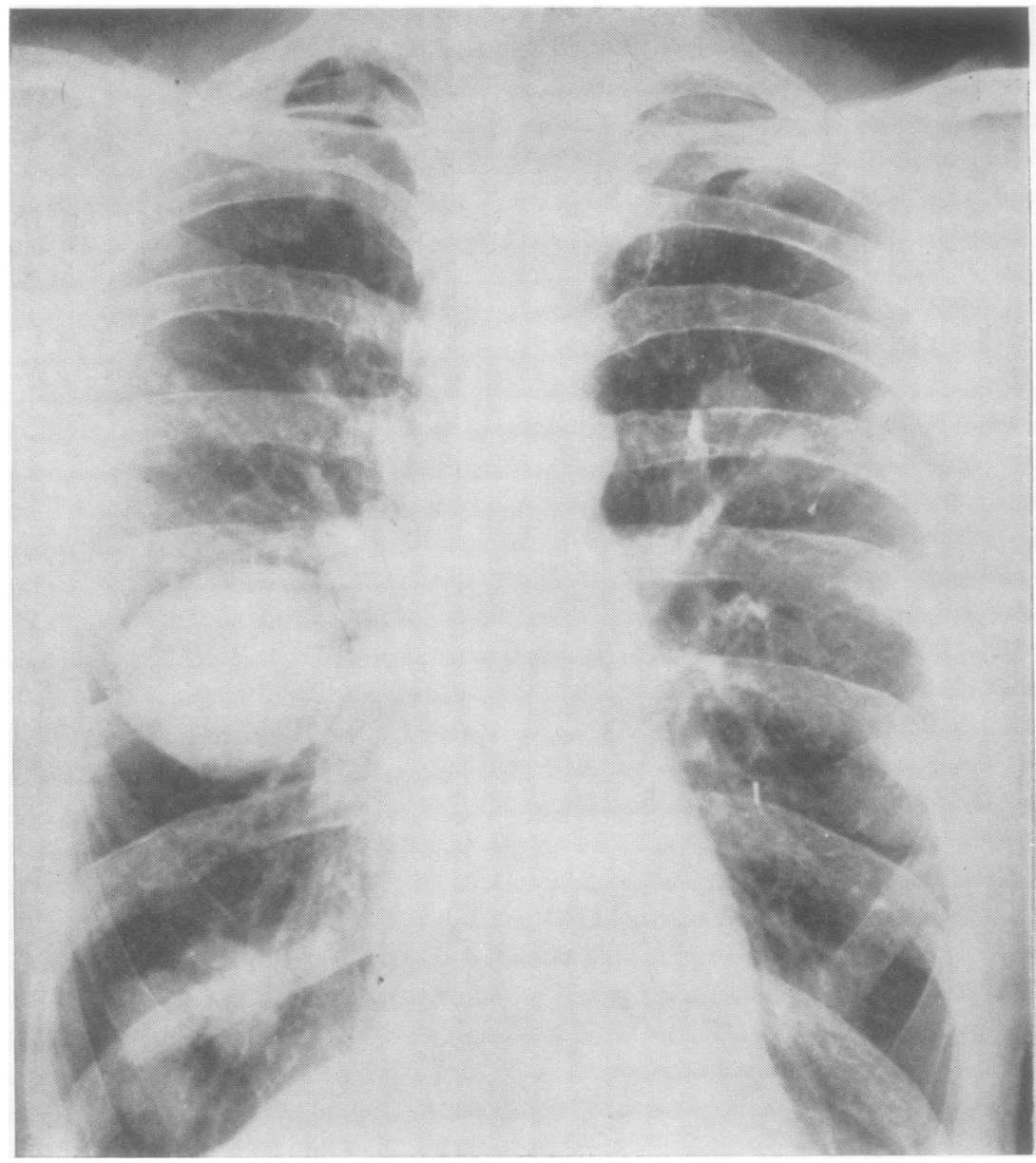

Fig. 1a

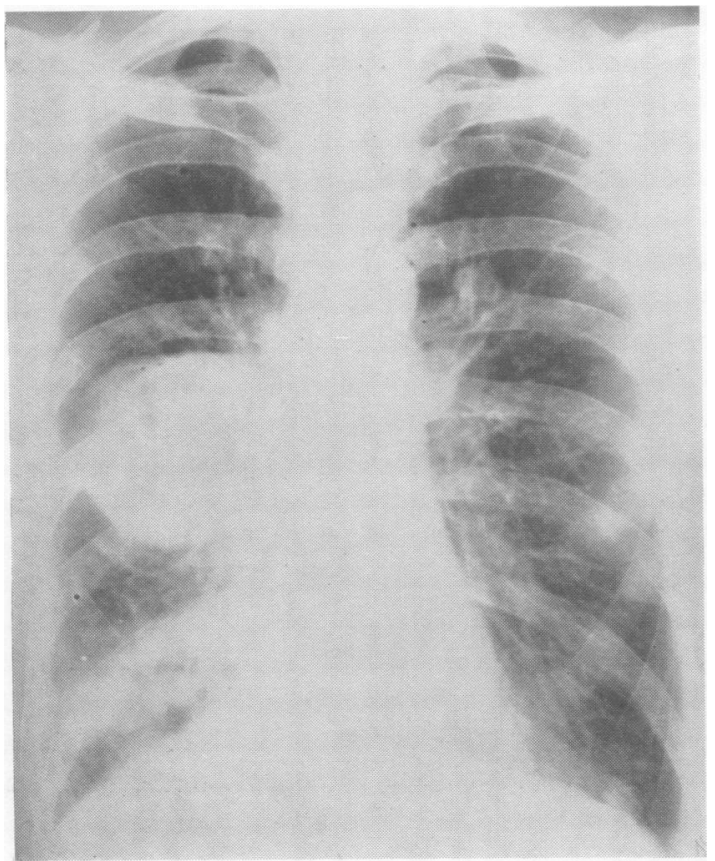

Fig. 1b

FIG. 1a.-Case 1: pulmonary metastases in 1938. four years a orchidectomy for seminoma.

FIG. 1b.-Case 1: appearance of metastases in 1940.

Fig. 1c.-Case 1: calcification in the metastases in 1948.

FIG. 1d.-Case 1: large cavity with fluid level in apex of lower lobe (1948).

Fig. 1e.-Case 1: unchanged appearance of calcified metast in 1950. 


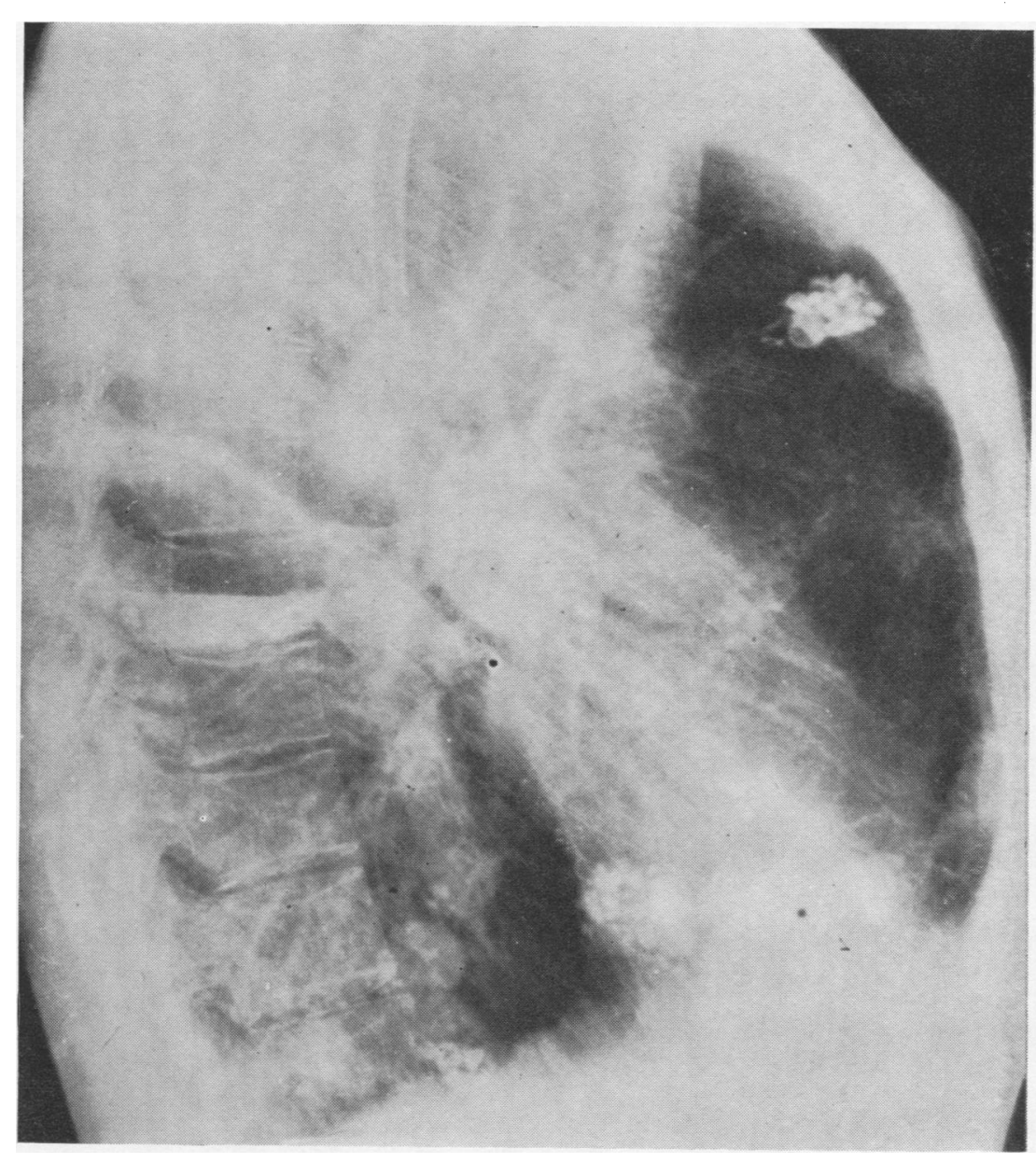

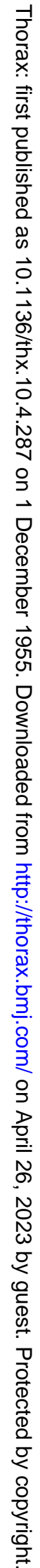

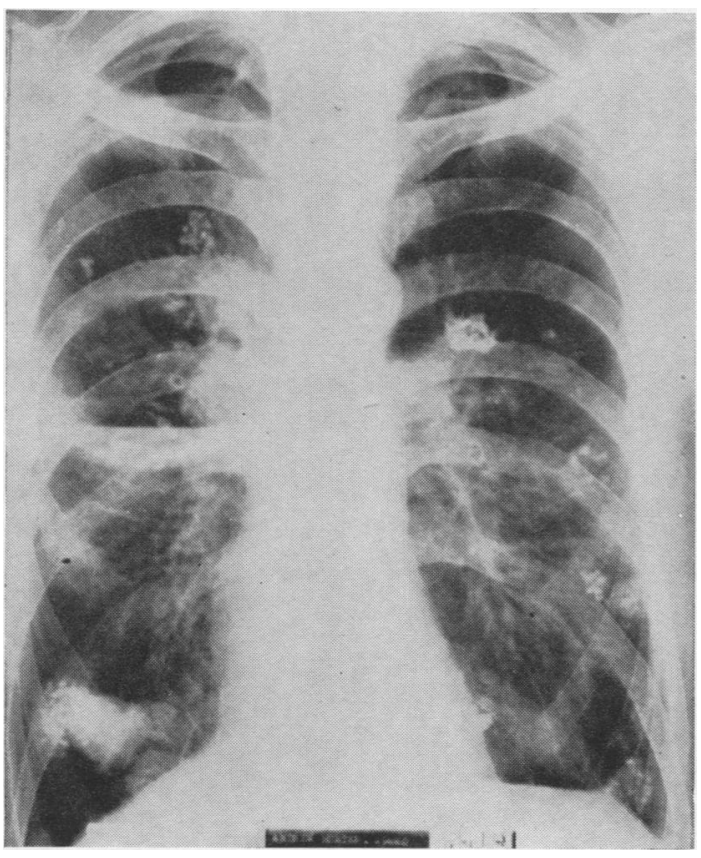

Fio. 1c

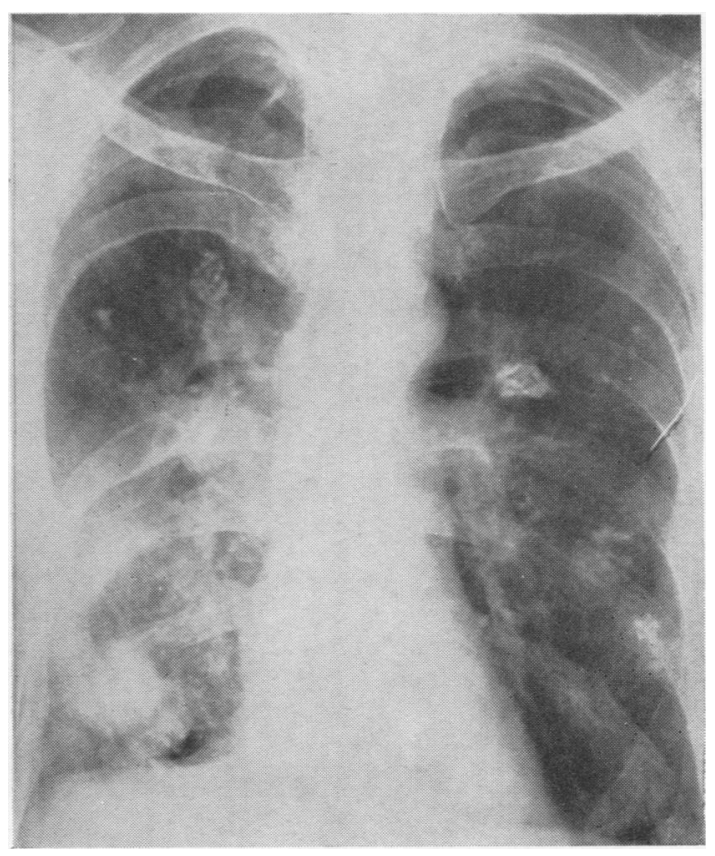

Fig. 1e 


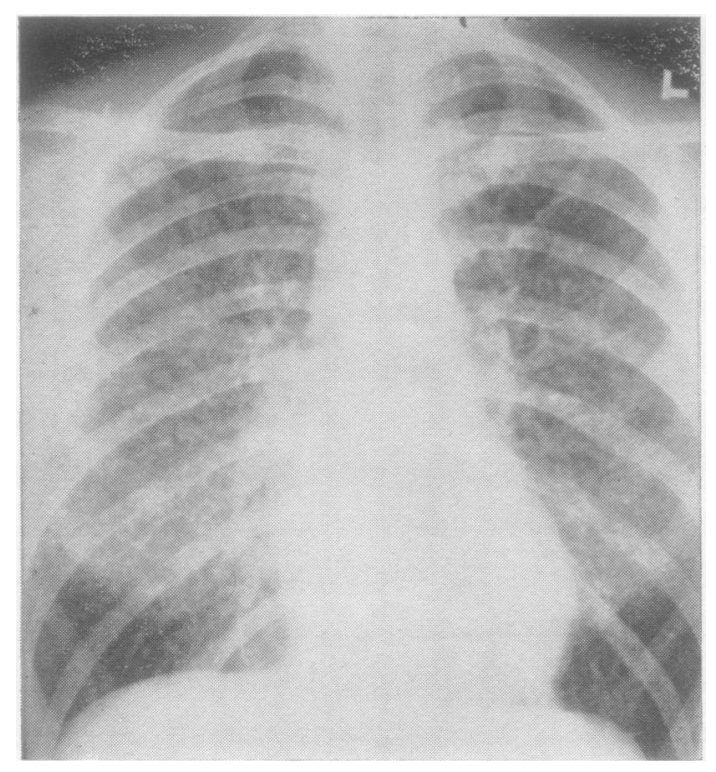

FIG. 2a

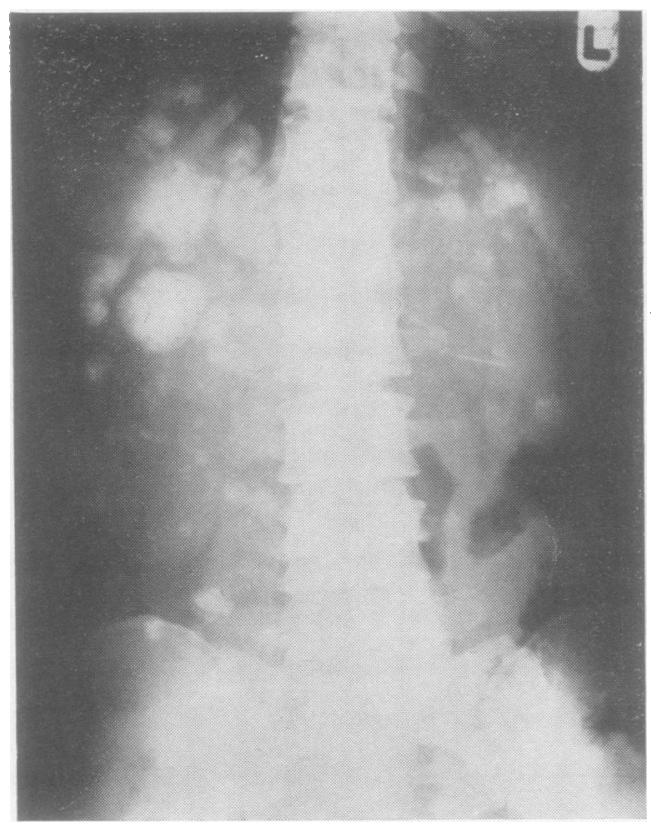

FIG. 2c

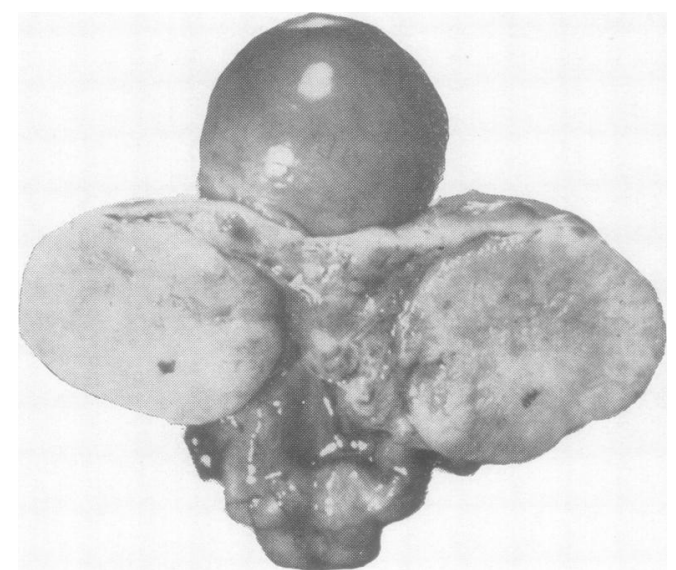

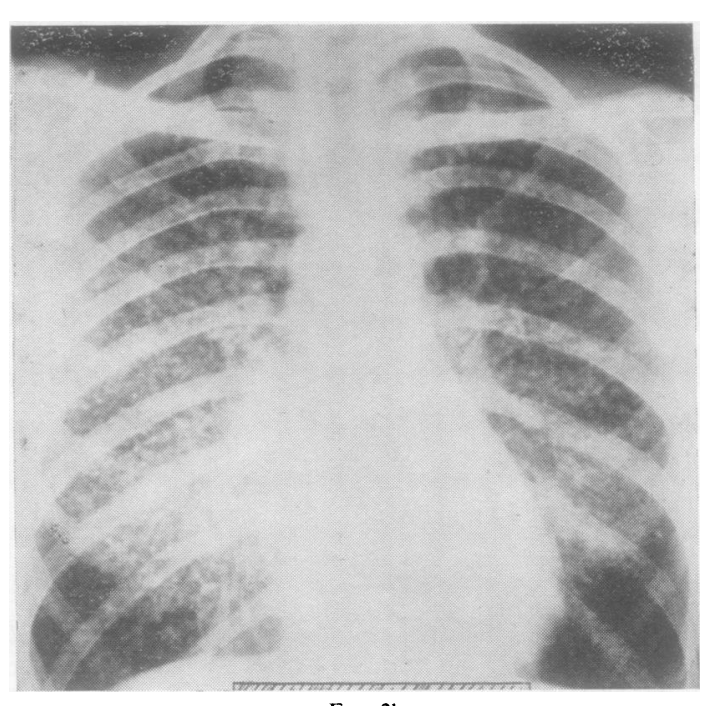

Fig. 2b

S.

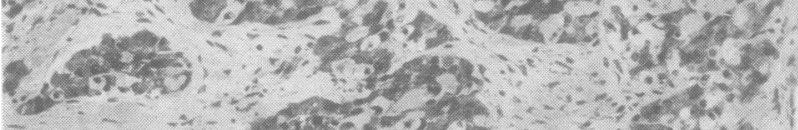

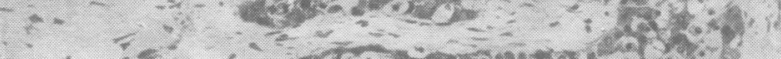

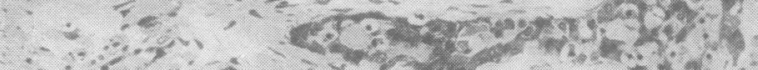

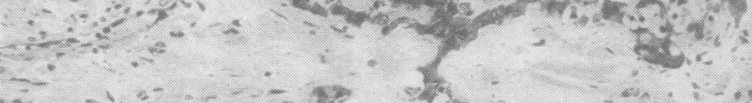

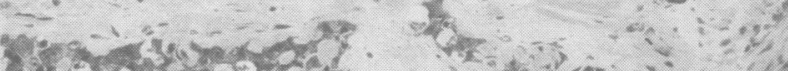

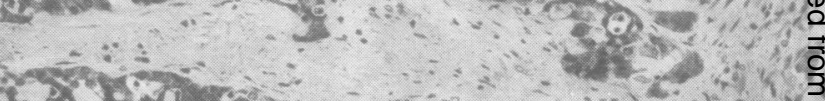
4.

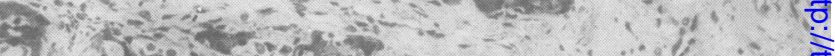

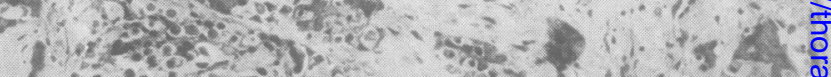

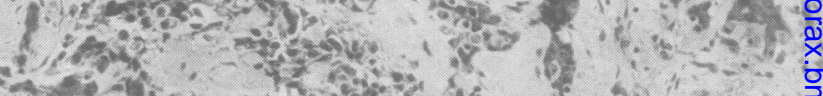

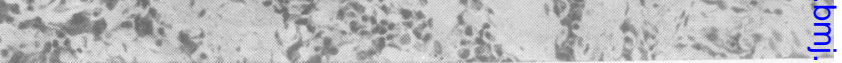
FIG. 2e ○े 25. 
being negative for tubercle bacilli, and the Mantoux reaction negative at 1 in 100 dilution. A 60-day course of streptomycin with P.A.S. was given and she was subsequently discharged with the possible diagnosis of chronic miliary tuberculosis or sarcoidosis.

During 1951 there was a little loss of weight, together with mild attacks of diarrhoea and unproductive cough. In July, 1951, she was again admitted to a hospital, where, in the course of barium enema examination, numerous calcific opacities were noted in the abdominal radiographs. A cervical lymph node biopsy was reported as showing "typical epithelioid tubercles of sarcoid." In December, 1951, on her final admission to hospital, she was complaining of lassitude and slight cough, and also amenorrhoea of four months' duration. Physical examination revealed a thin woman in fairly good general condition. Small, firm cervical lymph nodes were palpable and the liver was enlarged $5 \mathrm{~cm}$. below the costal margin.

The tuberculin reaction was positive (1 in 100 dilution) and the gastric juice repeatedly negative on culture for M. tuberculosis. The cellular and chemical components of the blood were normal. The chest film showed generalized small pulmonary opacities (Fig. 2b), a picture essentially unchanged since February, 1950, except that the opacities were "harder" and probably calcified. A radiograph of the abdomen revealed multiple rounded calcified opacities lying anteriorly over a wide area (Fig. 2c).

In January, 1952, a laparotomy was performed with the intention of removing one of these calcified masses, the diagnosis of sarcoidosis being considered untenable. The liver was found to be enlarged to below the umbilicus and was studded throughout with white nodules, varying in size, some showing central umbilication. One of these was removed. The right ovary was cystic and the left was represented by a cyst together with a round tumour the size of a billiard ball (Fig. 2d). Th:s ovary was removed.

Histological examination of the left ovarian tumour (Fig. 2e), the hepatic nodule (Fig. 2f), and the previously excised lymph node (reported erroneously as " sarcoid") showed "round-cell scirrhous ovarian carcinoma, of slow growing type, provoking intense fibrosis with granules of calcification. In many places

Fig. 2a.-Case 2: radiograph of February, 1950, showing " miliary" pulmonary opacities.

FIG. 2b.-Case 2: probable calcification in the pulmonary oracities.

FIG. 2c.-Case 2: radiograph of the abdomen showing multiple calcified hepatic metastases.

Fig. 2d.-Case 2: left ovarian tumour.

Fig. 2e.-Case 2: ovarian tumour with abundant fibrous stroma (haematoxylin and eosin. $\times 110$ ).

FIG. 2f.-Case 2: liver showing tumour with areas of calcification (haematoxylin and eosin. $\times 96$ ). the growth shows evidence of being strangled by fibrous tissue."

The patient made an uneventful recovery and was discharged from hospital in February, 1952. Seen at intervals since, she has remained reasonably well, and in 1955 radiological examination of the chest and abdomen shows little change.

\section{Discussion}

Case 1 was found to have cannon-ball lung metastases four years after removal of a testicular tumour. Eleven years after this operation, stippled calcification was seen in the deposits. The largest secondary tumour discharged its contents into a bronchus 13 years after operation, producing a large cystic cavity. This cystic tumour was removed at thoracotomy and was found to have the histological characteristics of a teratoma. The patient died 18 years after the removal of the primary testicular tumour, 14 years following the discovery of metastatic lung deposits.

The only comparable case we can find in the literature is described by Speed (1943), where a patient developed lung metastases 10 months after removal of an osteogenic sarcoma, but survived for 13 years with well-marked calcification of the secondary deposits. Zerman (1943) reported the death of a patient shortly after lung metastases had appeared $11 \frac{1}{2}$ years after orchidectomy for teratoma. Cavitation of a secondary deposit in the lung is in itself of unusual occurrence, having previously been reported by Farrell (1935) in a patient with metastatic sarcoma, by Minor (1950) in four instances in a series of 312 cases of metastatic pulmonary carcinoma, and by Salzman, Reid, and Ogura (1953), who described cavernous metastases from a seminoma of testis and from a carcinoma of pancreas. The last-mentioned writers ascribed such cavitation to rapid growth of the metastases, but in our case it appeared 10 years after radiological demonstration of the lung deposit.

Our Case 2 had calcified nodular metastases in the liver, involvement of lymph nodes, and calcified miliary deposits in the lungs from a primary ovarian carcinoma. A chest radiograph suggested chronic miliary tuberculosis or sarcoidosis, and a correct diagnosis was not made until two years later when a radiograph of the abdomen was taken and a laparotomy performed. Histological examination of the ovarian tumour, of a hepatic metastasis, and of a cervical lymph node all showed a picture of ovarian carcinoma. She is alive and well five years after the original discovery. 
Considering delayed growth of metastatic tumours, Willis (1934) stated that the majority of malignant tumours present a steady and uninterrupted course to a fatal termination, but with certain exceptions as follows:

(1) Tumours which, although frankly malignant in type and productive of metastases, exhibit long duration with little or no impairment of the patient's health.

(2) Tumours which, following surgical removal, recur locally only after a long period of freedom from disease.

(3) Tumours which, in spite of completely successful local treatment followed by a long period of health, recur as metastases.

(4) Tumours which exhibit spontaneous retrogression, partial or complete, in their primary or metastatic situation.

Our two patients fall into Group 1 of this classification, and would seem of sufficient interest and importance to report.

\section{SUMMARY}

Two patients are described, one with a primary testicular teratoma, the other having a primary ovarian carcinoma, each associated with calcified metastases.

The male patient lived 18 years after orchidectomy, and 14 years after discovery of the lung metastases. The woman is alive five years after a chest radiograph showed disseminated carcinomatosis.

The largest of the teratomatous metastatic deposits became cavernous 10 years after it appeared, and was then resected.

We would like to thank Mr. Bruce M. Dick for permission to publish Case 1 .

\section{REFERENCES}

Farrell, J. T. (1935). Radiology, 24, 444

Minor, G. R. (1950). J. thorac. Surg., 20, 34.

Salzman, E., Reid, J. H., and Ogura, G. I. (1953). Dis. Chest, 23, 678

Speed, K. (1943). Surg. Gynec. Obstet., 76, 139.

Willis, R. A. (1934). The Spread of Tumours in the Human Body. Churchill, London.

Zerman, P. (1943). Med. J. Aust., 2, 315. 